\title{
Crónica histórica de ACTAS UROLÓGICAS ESPAÑOLAS en su XXX aniversario
}

\author{
Pérez Albacete M. \\ Hospital Universitario Virgen de la Arrixaca. Murcia
}

Actas Urol Esp. 2007;31(4):299-315

\section{RESUMEN}

\section{CRÓNICA HISTÓRICA DE ACTAS UROLÓGICAS ESPAÑOLAS EN SU XXX ANIVERSARIO}

Objetivo: Con motivo de su trigésimo aniversario pretendemos conocer, desde el punto de vista histórico, el desarrollo y la evolución de Actas Urológicas Españolas. Para ello analizamos el papel desempeñado por cada uno de los directores que la revista ha tenido en este periodo.

Material y métodos: Revisamos el contenido de la publicación desde enero de 1977 a octubre de 2006 , el título quinto de los estatutos de la Asociación Española de Urología que la regulan, sus diversas modificaciones y, también, cuantas noticias aparecen en relación con la revista en el Boletín Informativo de la AEU.

Resultados: Detallamos la actuación de cada director y señalamos las medidas tomadas, los cambios y las adaptaciones efectuadas para mantenerla al dia y para situarla dentro del contexto internacional de las revistas biomédicas, su inclusión en los índices internacionales de medida y en Internet, con la finalidad de incrementar su difusión, el factor de impacto y la intención de consolidarla como la de referencia urológica entre las de habla hispana.

Conclusiones: La revista ha recogido fielmente la labor de los urólogos españoles y ha sido el exponente en estos treinta años de la evolución y del progreso constante que ha mantenido nuestra Urología. Es patente, en sus 30 volúmenes, la entrega de sus cuatro directores, la continua preocupación y la lucha que sostienen por superar las dificultades y elevar su calidad científica para alcanzar los objetivos propuestos.

Palabras clave: Historia de la Urología. Urología española. Revistas de Urología. Publicaciones periódicas. Actas Españolas de Urología.

\section{ABSTRACT \\ XXX ANNIVERSARY OF ACTAS UROLOGICAS ESPAÑOLAS: HISTORICAL CHRONICLE}

Objective: In the occasion of his thirtieth anniversary we try to know, from the historical point of view, development and evolution the Spanish Actas of Urology. For it we analyzed the role played by each one of the directors who the magazine has had in this period.

Material and methods: We reviewed the content of the publication from January of 1977 to October of 2006, the title fifth of the statutes of the Spanish Association of Urology that regulate it, its diverse modifications and, also, whichever news appear in relation to the magazine in the News bulletin of the AEU.

Results: We detailed the performance of each director and indicated the measures taken, the changes and the conducted adaptations to maintain it to the day and to locate it within the international context of the biomedical magazines, its inclusion in the international indices of measurement and Internet, with the purpose of increasing its diffusion, the factor of impact and the intention to consolidate it as the one of urological reference between those of Hispanic speech.

Conclusions: The magazine has gathered faithfully the work of the Spanish urologist and has been the exponent in these thirty years of the evolution and the constant progress that our Urology has maintained. He is clear, in his 30 volumes, the delivery of his four directors, the continuous preoccupation and the fight that maintain to surpass the difficulties and to elevate their scientific quality to reach the proposed objectives.

Keywords: History of the Urology. Spanish Urology. Magazines of Urology. Periodic publications. Spanish Acts of Urology. 


\section{OBJETIVO}

Con ocasión del trigésimo aniversario de Actas Urológicas Españolas consideramos oportuno realizar una revisión histórica del desarrollo y de la evolución que ha llevado. Dado el papel fundamental que desempeña el director de la revista, como responsable de la edición, de la evaluación de su contenido, del orden y de la inclusión o no de notas o de artículos, de los fallos y de los aciertos que presente, creemos que la valoración debe recaer en conocer paso a paso los dados por su principal valedor, de ahí que dirijamos nuestros trabajo a seguir y detallar la actuación, por riguroso orden cronológico, de cada uno de los directores que la publicación ha tenido en estos años.

\section{MATERIAL Y MÉTODOS}

Como órgano de difusión de la Asociación Española de Urología (AEU) revisamos en sus estatutos el título quinto, que es el que regula su funcionamiento, y las diversas modificaciones realizadas a lo largo de estos años. Los 244 números editados desde enero de 1977 a octubre de 2006, incluidos los monográficos, tras el análisis entresacamos todas las referencias que sobre la publicación y las diferentes declaraciones de sus directores encontramos, además de repasar el Directorio y Reglamento que cada año se entrega en el Congreso Nacional de Urología, desde 1996, y también extraemos del Boletín Informativo de la Asociación, cuantas noticias hacen referencia a la revista y, por último, de la actas de la AEU, los informes preceptivos del director de Actas Urológicas en la asamblea general anual.

\section{RESULTADOS}

Bajo la presidencia, en la Asociación Española de Urología, del Dr. D. Juan Montero Gómez, durante la asamblea general ordinaria en el XL Congreso Nacional celebrado en Granada el 12 de junio de 1975 se aprueba dar un voto de confianza a la Junta Directiva para realizar todas las gestiones necesarias con el fin de cumplir el artículo segundo del reglamento que dice: La revista Actas Urológicas Españolas será el Órgano Oficial de la Asociación que refleje las actividades científicas de la misma. El 6 de julio de 1976, en León, en el transcurso del XLI Congreso, la asamblea aceptó por unanimidad la puesta en marcha de la publicación y el nombramiento del Dr. D. Carlos Alférez Villalobos como su director y facultó a la Junta de la AEU para la designación de un adjunto a la dirección y para la elección de los miembros que compondrían los comités, el de redacción y el asesor de la revista, así como para la constitución de la comisión coordinadora y de sus competencias (Fig. 1). El 12 de noviembre de 1976, el pleno de la junta directiva, aprueba el reglamento que la rige, las normas para su edición y para su financiación ${ }^{1}$, así como el periodo de cinco años reelegible por otros cinco, para los cargos de director y de director adjunto para el que es nombrado el Dr. D. Francisco Javier Solé Balcells (Fig. 2).

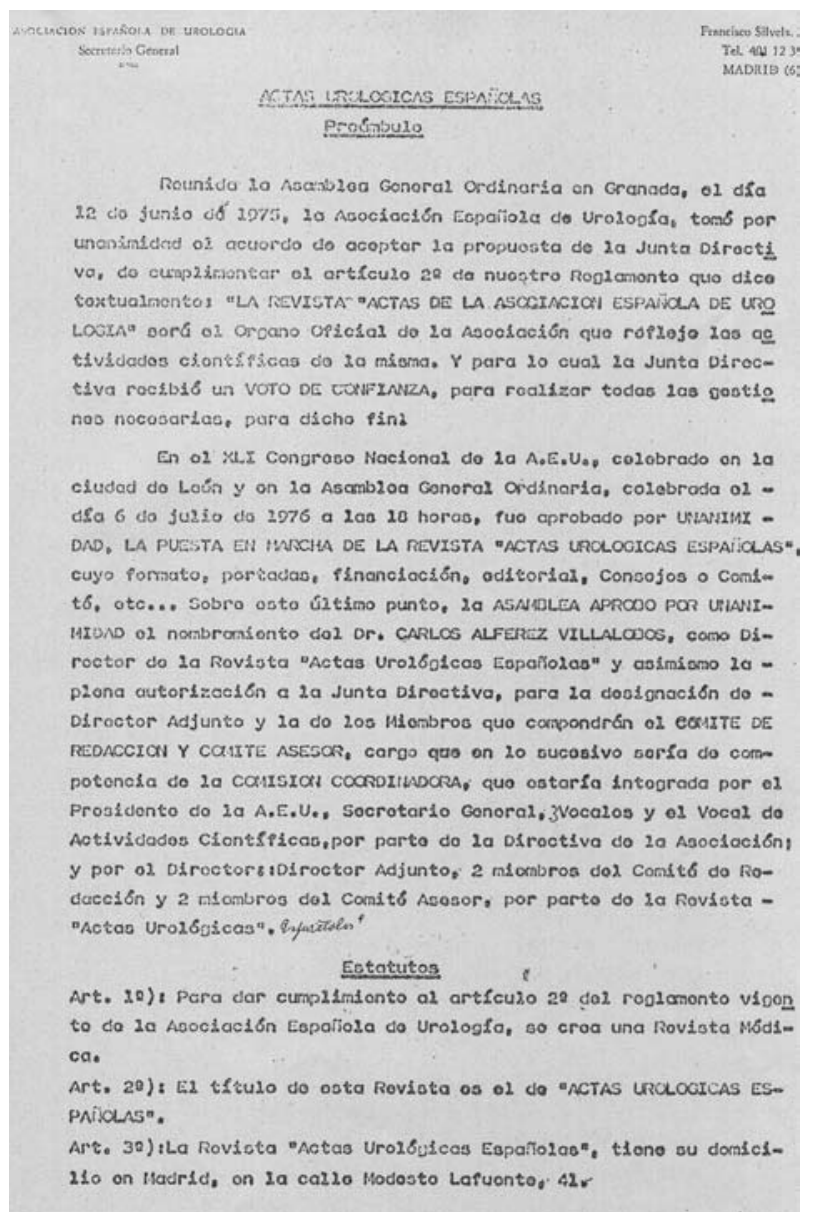

FIGURA 1. Acta de constitución de Actas Urológicas Españolas. 


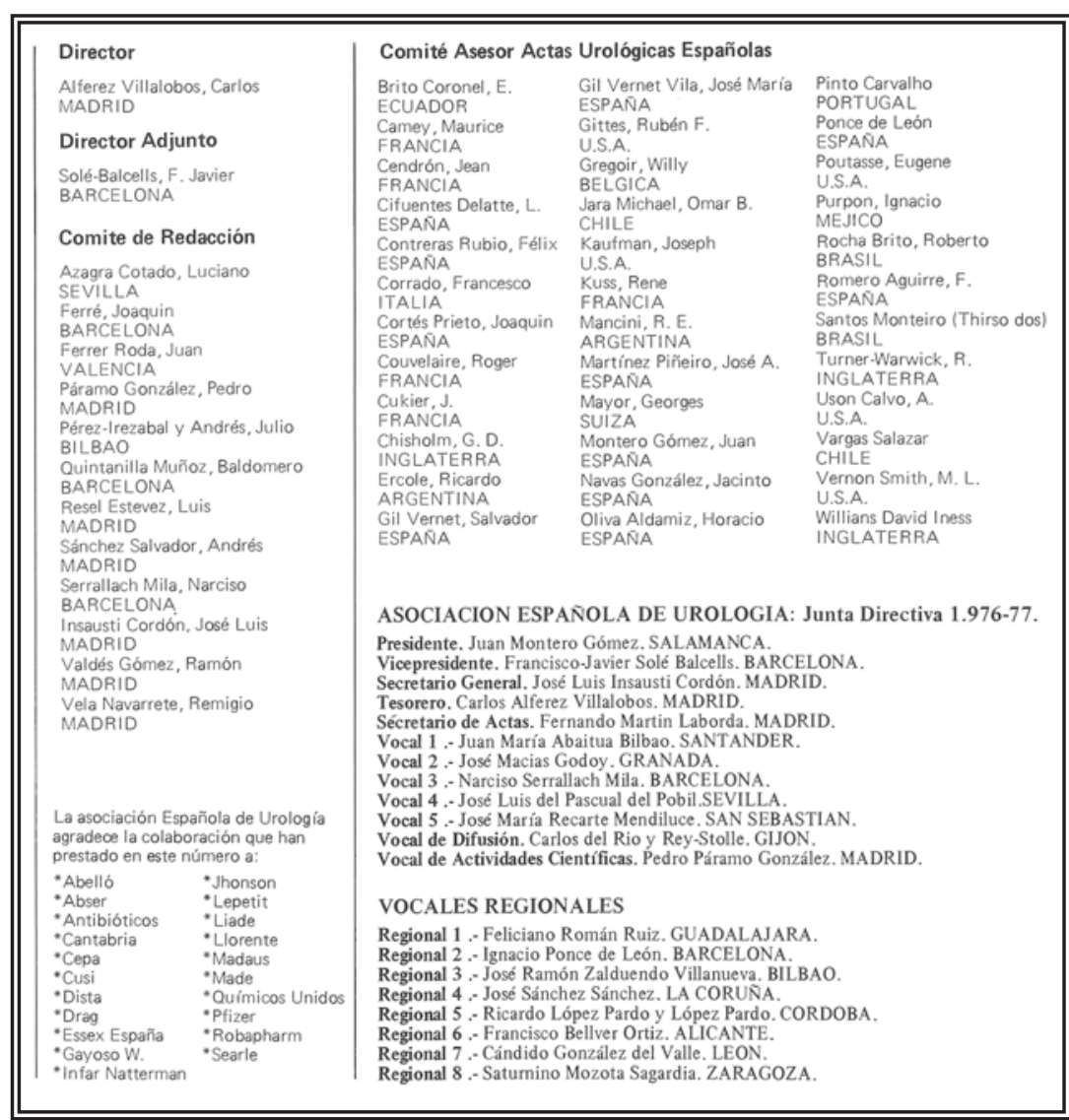

FIGURA 2. Primer Comité de Actas Urológicas Españolas.
El primer número ve la luz en enero de 1977, con una portada de color sepia en la que, bajo el título, centrado, de la revista y de la Asociación con letras de gran tamaño en negro, se recoge el anagrama de la Asociación, el número de orden que le corresponde y la fecha (Fig. 4), tiene una tirada inicial de 1.500 ejemplares; al abrirlo vemos reproducida la portada en pequeño tamaño en el ángulo superior izquierdo $\mathrm{y}$, debajo, los nombres de los directores seguidos de los componentes del comité de redacción, de las casas comerciales colaboradoras y el depósito legal (M4277-1977); en la segunda columna a la derecha, el precio, 900 Pts. ó 20 dólares USA, el domicilio social, la empresa editora, la de distribución, la de publicidad y la relación de miembros del comité asesor y de la Junta Directiva de la AEU, esta disposición sufrirá varias trasformaciones

\section{Dirección del Dr. Alférez (julio de 1976 a octubre de 1985). Vol. I (1) a X (2)}

Le cupo a Dr. Alférez (Fig. 3) la tarea de organizar y desarrollar el contenido de la revista, cuyas características técnicas, así como el sistema de su financiación por medio de la cuota de inscripción de los socios y la contribución de las casa comerciales, se encontraban recogidos en los estatutos; su distribución y su administración recaían en la sociedad Ediciones Nacionales Especializadas S. A. (ENE). Para su gobierno contaba la publicación con un comité de redacción, cuya misión era la de revisar los artículos que se remitian, y con un director que era quien daba la conformidad para editarlos; con un comité asesor que incluía personalidades de la Urología internacional, con unos veinticinco miembros de promedio, de los cuales son nacionales entre once y quince; el de redacción se encontraba coordinado por un vocal secretario elegido entre los doce urólogos españoles que lo componen, aunque su número variará a lo largo de los años hasta llegar a dieciséis.

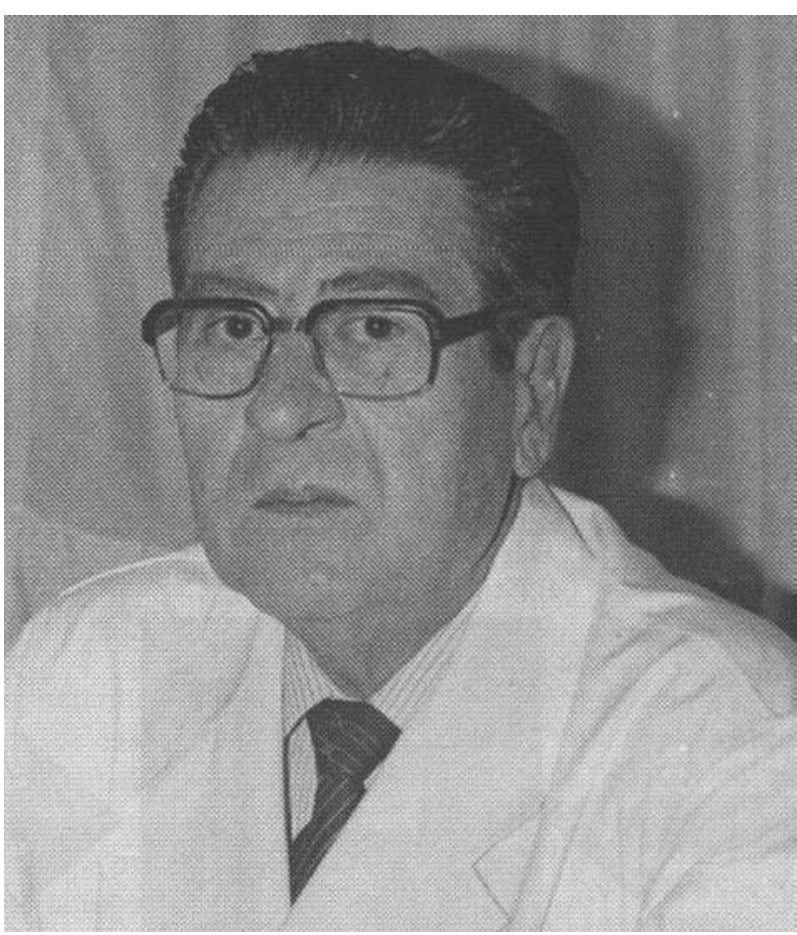

FIGURA 3. Dr. Alférez, primer director de Actas Urológicas (1976-1985). 


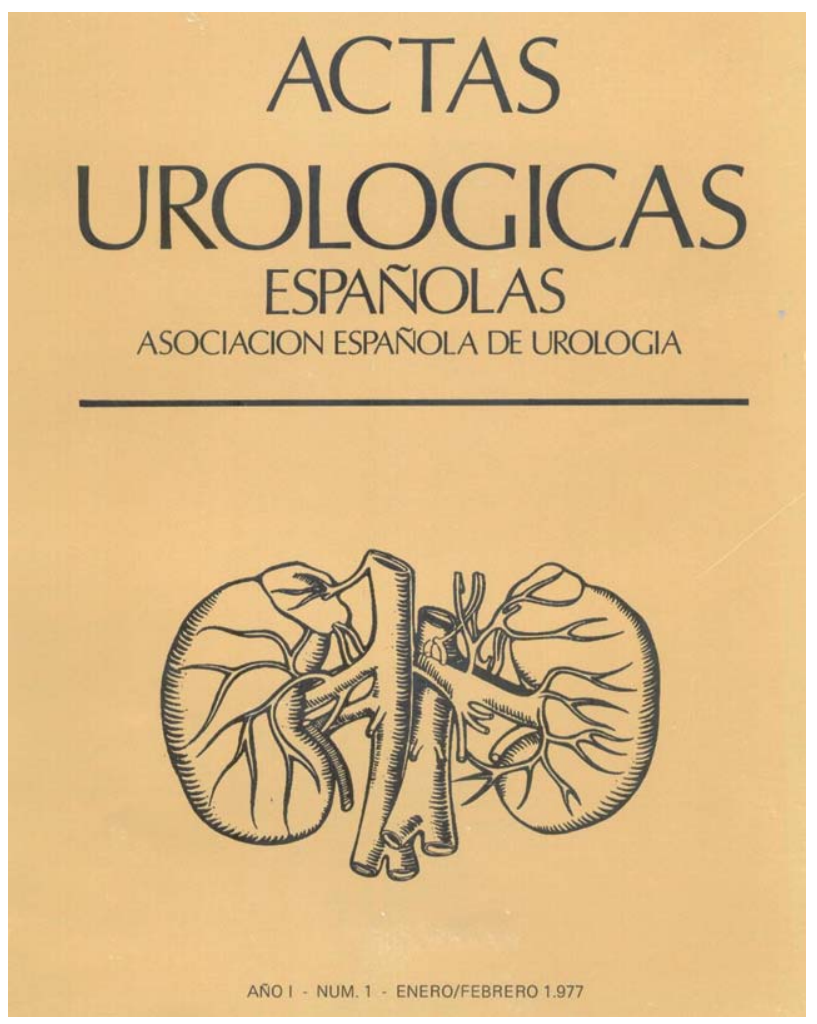

FIGURA 4. Primer número de Actas Urológicas Españolas, enero de 1977.

a lo largo de los años, la primera al mes siguiente. En septiembre de 1979 [Vol. III (5)], se incluye el Internacional System News Number (ISNN 0210-4806).

Comienza el contenido con unas palabras del Dr. D. Salvador Gil Vernet quien agradece el honor que se le hace y manifiesta su alegría por la edición, saluda a la comunidad urológica y declara que las escuelas de Urología que hay en España se encuentran a la altura de las de los países más avanzados pero que, además de su existencia, precisan de un medio en el que manifestar sus opiniones. La dirección justifica en un editorial la necesidad de contar con la publicación como cauce de expresión, el espíritu que la anima es pluralista y de amplios y variados conocimientos, criterios y opiniones con un solo objetivo, mostrar la enorme realidad de la Urología española ${ }^{2}$.

De periodicidad bimensual, incluye entre 70 y 100 páginas por número y un volumen por año que, en los nueve primeros, tiene un promedio de 407 páginas cada uno (entre 310 y 580); comienza por lo general con un editorial seguido de una comunicación especial o revisión de conjunto, entre cuatro y nueve artículos originales y unas cinco o seis notas clínicas por término medio y concluye con un apartado de noticias de la AEU, de los cursos que se organizan, del calendario de congresos y reuniones y de las normas para la admisión de los trabajos. Durante el primer año presenta el contenido a tres columnas (Fig. 5) y lo reduce a dos en el siguiente, formato que se mantiene hasta la actualidad; tras el título del artículo aparece el nombre de los autores y el del centro donde se ha llevado a cabo seguido de las palabras clave $\mathrm{y}$, al término del texto, se encuentra el resumen en español, francés e inglés; en cada página, en el borde superior izquierdo y con letra pequeña, se colocan el número y año correspondientes y en el derecho el nombre del responsable y el título del trabajo, esta distribución no variará hasta enero de 1989. Al final del año [vol. I (6)] incluye una separata, que realiza el Instituto de Información y Documentación en Biomedicina (CSIC) de la Universidad de Valencia (Fig. 6), con

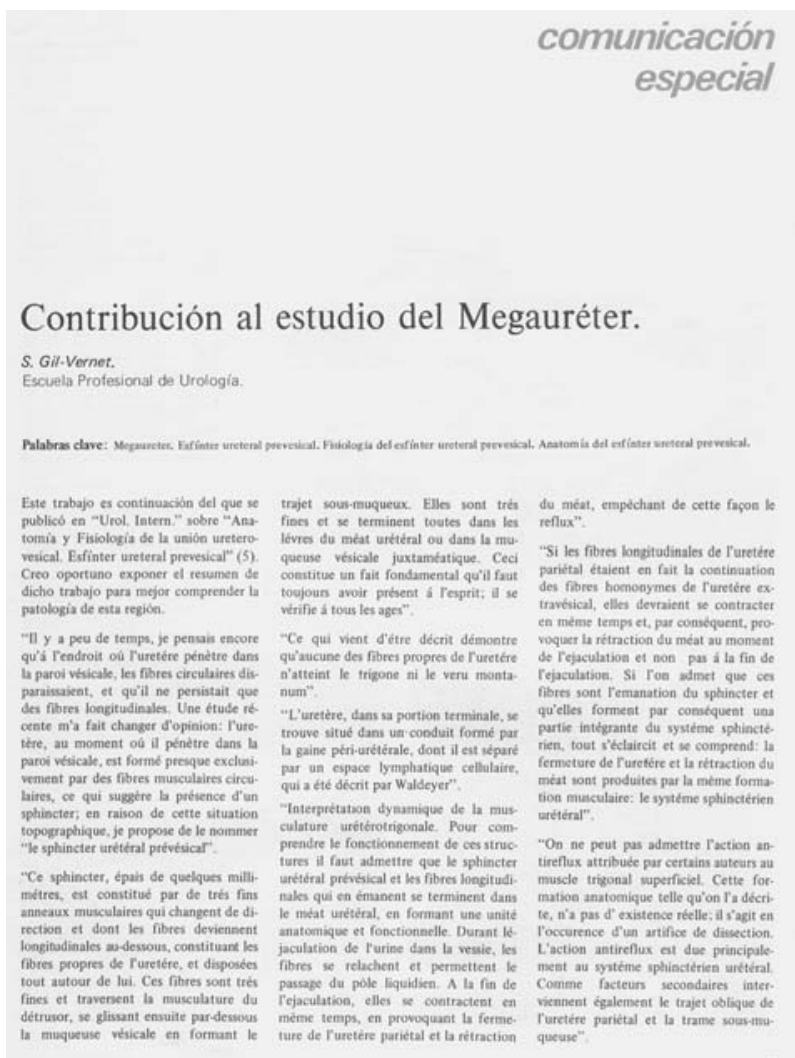

FIGURA 5. Primer artículo publicado del Dr. D. Salvador Gil Vernet, editado a tres columnas. 


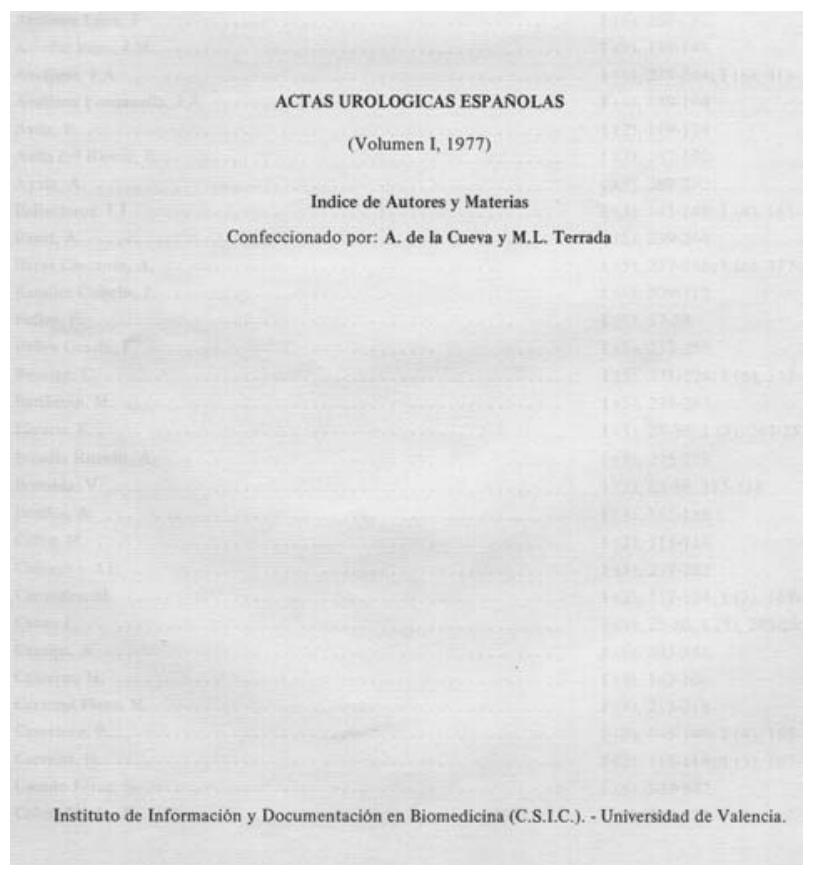

FIGURA 6. Índice de autores y materias del primer volumen, noviembre /diciembre de 1977.

el índice de los autores por orden alfabético y de las materias tratadas en el volumen que persistirá hasta 1986.

En mayo de 1982, al cumplir el periodo reglamentario, se prorroga la dirección del Dr. Alférez, cesa el Dr. Solé y el cargo de director adjunto recae en el Dr. D. José Luis Insausti Cordón, que permanecerá en él hasta diciembre de 1985, lo sustituirá por unos meses, de enero a abril de 1986, el Dr. Leiva. De esta segunda etapa señalamos la edición, en 1985, de un número monográfico con lo que la publicación se suma a la celebración del cincuentenario de la fundación de la $\mathrm{AEU}$, escrito por la pluma de los que nos precedieron, y que gracias a ellos la Urología española ocupa lugar destacado en la especialidad, en palabras del Dr. Alférez ${ }^{3}$.

En 1982 fue nombrada secretaria de la publicación $D^{a}$. María Jesús Arribas cargo en el que se mantiene hasta la actualidad; durante estos veinticuatro años ha sido y es la persona encargada de recoger los manuscritos y de velar por su curso durante el proceso editorial, para resolver o evitar cualquier problema surgido y lograr que cada número salga correctamente publicado en su momento, misión que ha desarrollado con esmero y gran eficiencia.

\section{Director del Dr. Leiva (mayo de 1985 a octubre de 1994). Vol. X (3) a XIX (1)}

En el L Congreso Nacional celebrado en el Puerto de la Cruz de la isla de Tenerife, en mayo de 1985, cesa reglamentariamente el Dr. Alférez y es propuesto por la Junta Directiva, con la aprobación unánime en el pleno de la asamblea plenaria, el Dr. D. Óscar Leiva Galvis como nuevo director. Asume el reto de trabajar para modernizarla, potenciarla y promocionarla dedicándole todo el tiempo que sea necesario. El cargo de director adjunto recae en el Dr. D. Narciso Serrallach Mauri, se instaura la figura del secretario de redacción para lo que es designado el Dr. D. Joaquín Carballido Rodríguez, cargo que no se renovará a su cese. El comité de redacción alcanza la cifra de veintiséis miembros y el asesor la de 45, de ellos veinte españoles.

En el número 3 de 1986, correspondiente a mayo / junio, figura el Dr. Leiva por primera vez como director; en enero de 1987 [vol. XI (1)] es manifiesto el cambio, la portada transforma el color sepia en blanco pero con idéntico contenido aunque usa el rojo para el anagrama y el negro para el nombre y la fecha; varía el tipo, el tamaño de letra y la calidad del papel, incluye el color en las microfotografías, introduce secciones como las revisiones de conjunto, las bibliográficas, resúmenes seleccionados de la literatura urológica internacional y la exposición de trabajos experimentales, incrementan los originales de diez o doce por número a veintitrés o veinticuatro, con lo que las páginas suben a 120; sustituye el índice anual coleccionable por el contenido de todo el año, distribuido por números, que se añade al número del mes de noviembre/diciembre ya desde 1986, [vol. X (6)]. En el de marzo/abril, la redacción informa de la necesidad de modificar las normas de admisión de manuscritos, con la finalidad de ajustarse a las medidas que el comité internacional editor de revistas biomédicas requiere, entre ellas el acuerdo del principio de responsabilidad del contenido del trabajo por todos los autores y, de este modo, darles uniformidad con la pretensión de elevar el nivel científico de la publicación ${ }^{4}$.

Aparece un número extraordinario sobre Oncología en 1987, se preocupa, la Asociación Española de Urología también, de impulsar el 
desarrollo científico y para ello establece los premios a los mejores originales editados durante el año en Actas Urológicas Españolas premios que entrega por primera vez en ese año, comunica el nombre de los ganadores en un segundo monográfico sobre infecciones urinarias en $1988^{5}$. Las ponencias de los temas monográficos presentados en el Congreso Nacional, que realiza la editora ENE con el soporte de la industria farmacéutica, a partir del LIV celebrado en Toledo en mayo de 1989, pasan a ser impresas con el logotipo de Actas Urológicas (Fig. 7) y continuarán publicándose sin modificaciones hasta el cambio congresual del año 2001 , en que desaparecen ${ }^{6}$; inicia también el Dr. Leiva la publicación del libro de resúmenes con el contenido de las comunicaciones expuestas en el congreso seguido por el índice alfabético de los autores, modelo que con mejoras cada año persiste hasta la actualidad (Fig. 8).
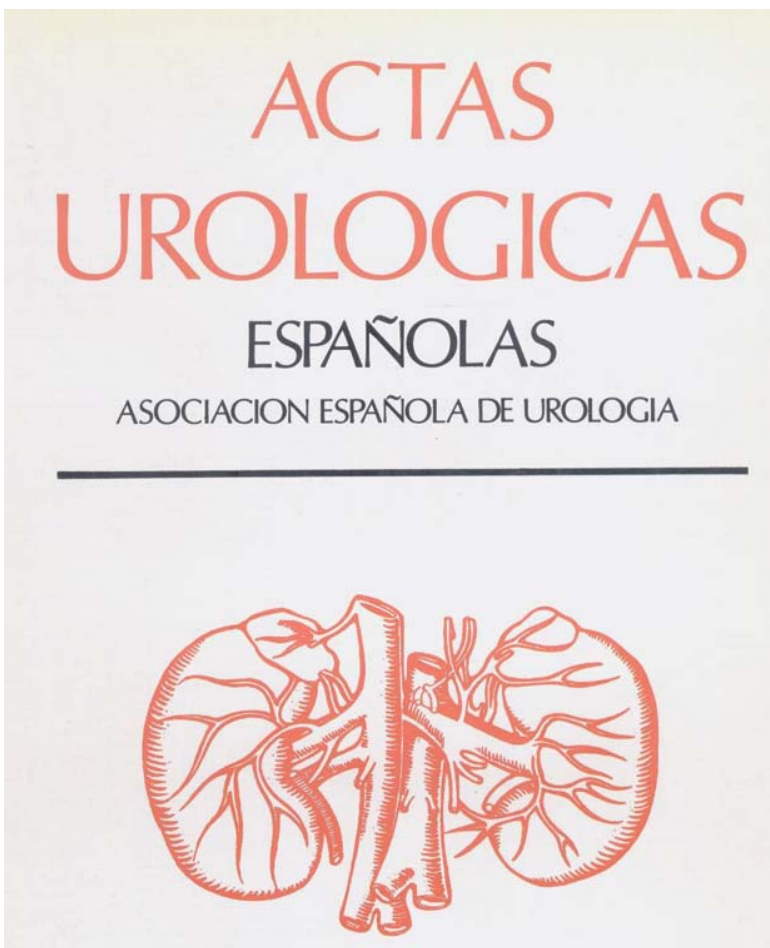

$$
\begin{aligned}
& \text { LIBRO DE RESUMENES } \\
& \text { LV Congreso Nacional de Urología } \\
& \text { Vigo, 24-27 de junio } 1990
\end{aligned}
$$

FIGURA 7. Primer libro de Resúmenes de los Congresos Nacionales, 1990, con el formato de portada modificada por el Dr. Leiva.

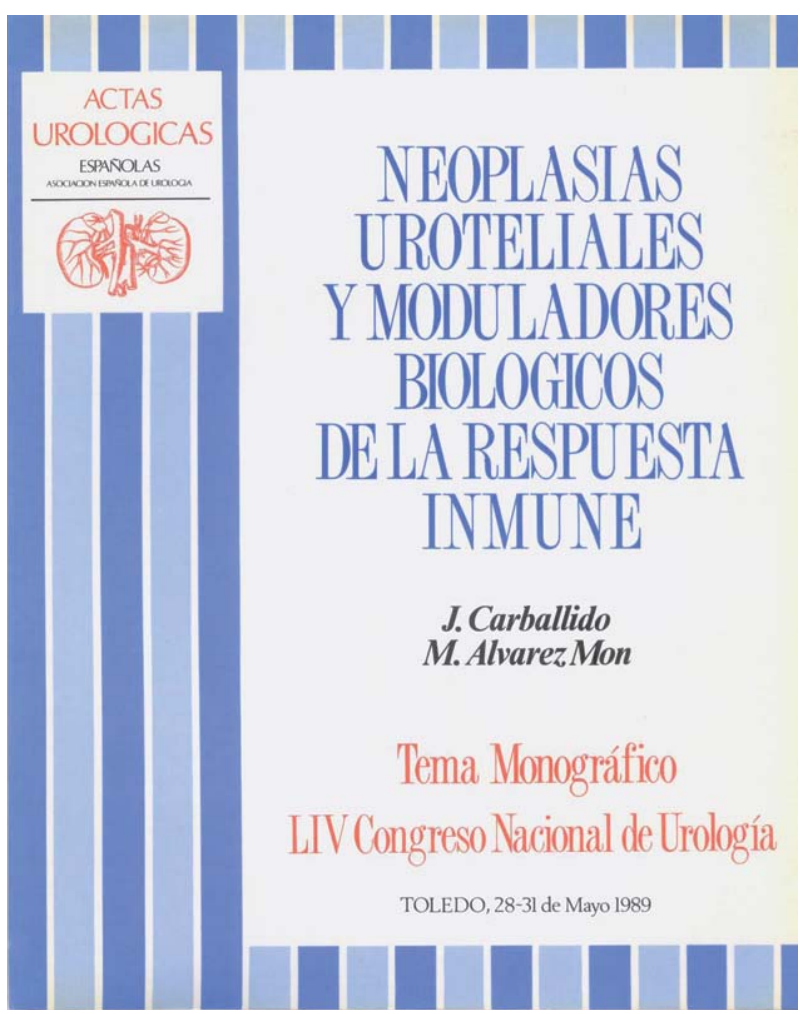

FIGURA 8. Portada del tema monográfico del LV Congreso Nacional de 1990 celebrado en Vigo.

En la reunión de la comisión coordinadora de Actas Urológicas, el 17 de noviembre de 1990, se acuerda renovar en la dirección a los Drs. Leiva ${ }^{7}$ y Serrallach. A partir de enero de 1991 [vol. XV (1)], incluye en la portada, en su parte inferior, por detrás del anagrama de la Asociación, cuatro bandas de color azul que paulatinamente se degrada. La primera manifestación escrita del director en su nueva etapa no llega hasta septiembre/octubre de ese año, en forma de carta en la que, además de agradecer el esfuerzo realizado por todos los implicados, señala que se incrementarán a diez los números anuales a partir de enero de 1992 y que se le dará una periodicidad mensual (excepto agosto y diciembre); con ello se podrá dar salida al cuantioso material remitido para su publicación ante el malestar existente entre los autores debido al importante retraso que lleva la aparición de los originales ${ }^{8}$.

En enero de 1992 [vol. XVI (1)] cambia el formato de la primera página en la que se encuentran reseñados los índices de consulta en los que se ha incluido la publicación: Excerta Médica, Índice Médico Español y el Index Médicus, junto 
con los datos de la sede social de la editora ENE, que se traslada de local en estas fechas, el resto del contenido, la composición de los comités y de la Junta directiva de la AEU, pasa a la segunda hoja.

En junio de 1993 se edita un número monográfico, con el directorio de los miembros de la Asociación en el que se añade el nuevo reglamento de la AEU, aprobado en asamblea extraordinaria en Madrid el 15 de abril de 1989 y ratificado el 28 de abril de 1990, en el que se regula la misión de la comisión coordinadora. El Dr. Vela, secretario general de la AEU, justifica la presentación como un especial de Actas Urológicas ya que tiene la ventaja de una mayor facilidad de consulta y permite conservarlo mejor al llevar el mismo formato que la revista $y$, además, sirve de vehículo para promover y mejorar el nivel de comunicación de todos los miembros de la asociación ${ }^{9}$. (Fig. 9). Los Dres. Leiva y Carballido hacen un llamamiento a todos los urólogos para que envien artículos ya que se ha logrado disminuir el periodo de espera para su edición y apro-

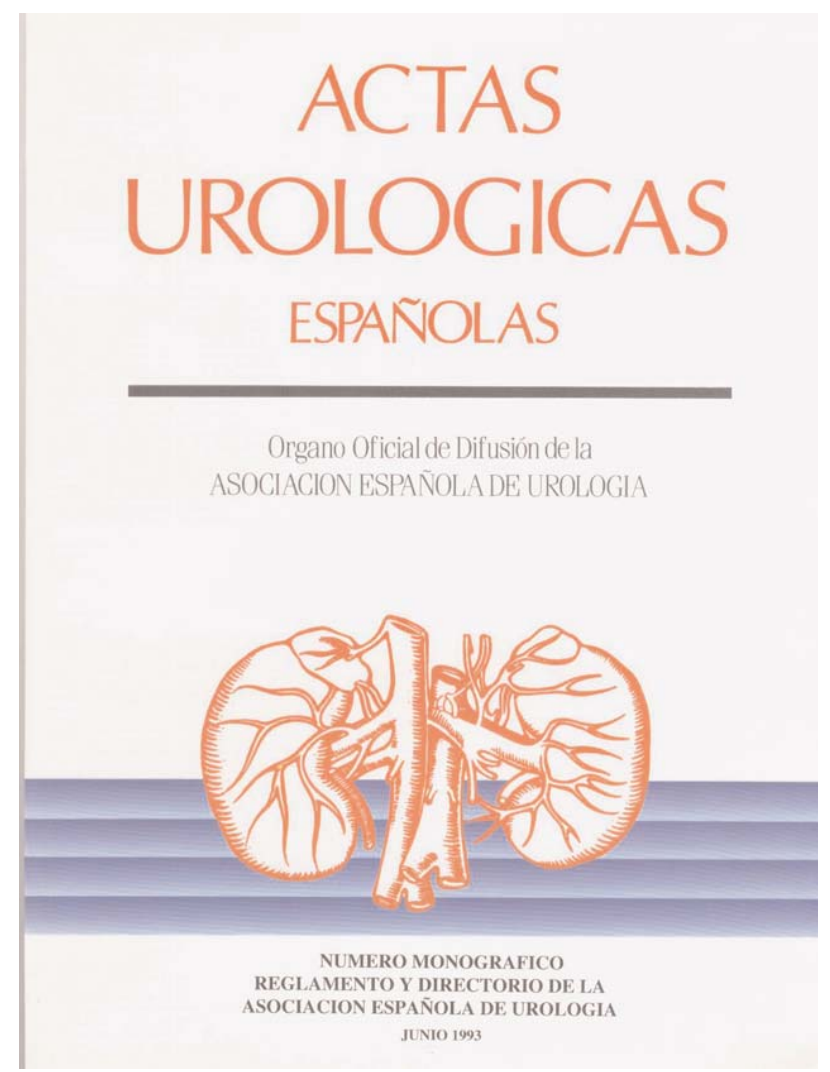

FIGURA 9. Primer Reglamento y Directorio de la AEU, junio de 1993. vechan para agradecer el esfuerzo que realizan los componentes del comité de redacción por su papel en la valoración y en el análisis de los originales remitidos, misión que consideran dura, difícil y que requiere gran dedicación de tiempo ${ }^{10}$.

En la misma fecha aparece un primer estudio sobre el contenido de la revista, los Dres. Blasco y Pinsach estudian la bibliografía de los editados en Actas Urológicas Españolas entre 1989 y 1990, en relación a la frecuencia con que se encuentran citados trabajos nacionales ya sea de libros, revistas, tesis o congresos, y hallan que únicamente alcanzan un 14,42\% del total, si descuentan las comunicaciones a los últimos, disminuye el número al 10,9\%; señalan como posible causa de esta baja utilización, la dificultad de localizar los artículos al no estar introducidos en los indices internacionales de consulta ${ }^{11}$.

Desde el mes de abril y hasta noviembre/ diciembre de 1994 se incluye una nota de la dirección de la AEU mediante la cual se adhieren a la resolución de la asamblea de la Organización Médica Colegial, de 7 de julio de 1993, en relación con los medios de comunicación y la ética, con una decidida voluntad de defender la libertad en general y la de la profesión médica en particular... por ello la junta directiva quiere ser la garante moral de que la ética de sus miembros, en materia de su publicidad, sean las que marcan las normas deontológicas ${ }^{12}$.

En mayo de 1994 edita un número monográfico como homenaje a todos los miembros que han pertenecido al comité de redacción, por su labor desinteresada y de gran rigurosidad con la que han contribuido al mantenimiento del prestigio de la revista. En él se despide el Dr. Leiva de la dirección, con un articulo titulado $\mathrm{El}$ deber cumplido (Fig. 10) en el que agradece a los que lo apoyaron y confiaron en él para dirigirla y a la editorial por su esfuerzo en llevar a cabo todos los cambios propuestos sin que las mejoras realizadas hayan impedido conservar su autosuficiencia económica (13). Junto con el Dr. Carballido nos ofrecen en el mismo número un interesante informe sobre el funcionamiento interno de la publicación, que permite conocer la metodología utilizada por la dirección en el tratamiento y valoración de los textos, desde enero de 1977 hasta diciembre de 1991; en estos 15 años los artículos 


\section{EL DEBER CUMPLIDO}

Cuando en una trayectoria profecsional, se rememofan las principales efemérides el nombramiento como DIREC TOR CIENTIFICO de la Revista ACTAS UROLOGICAS ESPAÑOLAS, Organo Oficial de Difusión de la Asociación Española de Urologia, fue para mí hasta ese momento, una de niis mayores alegrias y a la vez uno de los mayores retos, que se me habian planteado, al convertimne en responsable directo de orientar y dirigir ésta y mestigiosa revista, que por aquel entonces y des de la fundación de la misma en 1977 dirigia con acieno y sabidur mo de los urólogos que más han contribuido al engrandecimiento de la propia Asociación y de la Urologia nacional. it Dr. Carlos Alférez Villalobos, al cual siempre le he tenido infinito respeto $\mathrm{y}$ agradecimiento por haber sido un

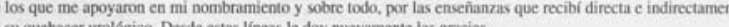

Tambien fueron responsables de mi nombramiento, la Junta Directiva, encaberada por el gran "Patriarca" Tr. José Luis Insausti Cordón y los Drs. Antonio Cimadevila Covelo. Narciso Sernallach Mila, Luis Rese

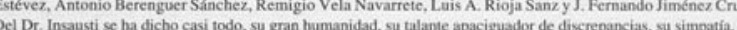
bel Dr. Insaust ise ha dicho casi todo, su gran humanidad, su tulante apaciguador de discrepancias, su simpatia, su osionados con aportar trabajo y honestidad a la Asociación Española de Urología dentro de los cuales me incluyo. Para ef mi mis emocionado recuerdo.

El cambio en la responsabilidad de dirigir a la Asociación, por el Dr. Luis A. Rioja Sanz, fue tambien para mí. otro expaldarzzo en la confianza que e y su equipo dinectivo me otorgaron, reflejado en la reeleccion por otro period. Aparte de la amistad siempre demostrada por el, su decidido apoyo fue muy importante para la dirección de
Revista.

Una vez tomada posesí́n del cargo, empezó para mí, el potenciar la publicación, para lo cual conté también con colaboración inestimable de la Editorial, la cual desde el principio no escatimo csfuctzos cr cuanto a

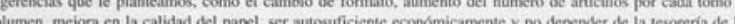
Asociación, compromisos que e e han cumplido hasta la fecha, motivos lo suficiemenente inupotentes eone per que tengan el reconocimiento de agradecimiento de toda la familia urologica.

En una etapa también temprana de mi responsabilidad, tuve la suerre de contar con el Dr. Joaquín Carballido Rodríguez corno colaborador al cual también le doy las más efusivas gracias por su aportación desinteresada, pero termendamente valiosa, en la planificación de todas las reformas que llevamos a cabo en la revista hasta alcan d "estatus" que tiene actualmente.

Quisiera también dar las gracias a todos los miembros de los Comités de Recheción y Asecor que trabujaron a

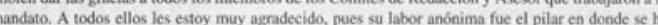
antentado la calidad de los trabajos y con sus correcciones y aportaciones han enriquecido la Revista.

El reto ha sido impontante, erro que trahujamos con honradea, ilusión desinteresodamente, pero se impone d elevo, no sólo por reglamento sino para que entren ideas renovadoras, se adapten neevas tendencias a la difusifo del pensamicnto cientifico español, que por su calidad y cantidad se merece una Revista de prestigio.

Me resta despedirme con estas lineas, de toda la comunidad urológica y pedir disculpas, por si alguien piensa que

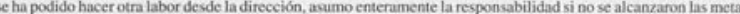
pero me voy con la conciencia trinquila del DEBER CUMPLIDO.

\section{Oscar Leina Galvis} Junio de 199

FIGURA 10. Despedida del Dr. Leiva, El deber cumplido, número monográfico de mayo de 1994, vol. XVII.

remitidos fueron 1.754 de los que se editaron 1.539, el 87,7\%, y fue rechazados por el comité de redacción el $12 \%$; el periodo medio de tiempo transcurrido entre el envío del original y su publicación fue de 7,8 meses (2-24); analizan también las referencias bibliográficas nacionales aparecidas en Actas Urológicas en lo que coinciden con las conclusiones del trabajo reseñado anteriormente, si bien lo justifican considerándolo como aceptable respecto a los patrones internacionales sobre consumo de información en la producción científica ${ }^{14}$.

El cese reglamentario del Dr. Leiva se produce en junio de 1994, pero continuará dirigiendo la revista "en funciones" hasta enero de 1995.

\section{Dirección del Dr. Carballido (octubre de 1994 a diciembre de 2003). Vol. XIX (1) a XXVII (10)}

En la asamblea ordinaria de la AEU celebrada en Sitges, bajo la presidencia del Dr. Resel Estévez, en mayo de 1994, en el LX Congreso Nacional, se establece una convocatoria abierta para la presentación de una memoria para renovar el cargo de director de Actas Urológicas Españolas. En la reunión de la comisión coordinadora, responsable de su elección, el 17 de octubre de 1994, es nombrado por unanimidad el Dr. D. Joaquín Carballido Rodríguez ${ }^{15}$ y, como adjunto, el vocal de actividades científicas Dr. D. Marceliano García Pérez quien, al cesar como responsable de la vocalía de actividades científicas de la AEU, es sustituido por el Dr. D. Carlos Rioja Sanz.

Llega el Dr. Carballido a la dirección de la revista con ilusión, con la inquietud de potenciarla y de aumentar el prestigio científico alcanzado. Con las reflexiones de su propia autocritica, fruto de su conocimiento de la publicación, acepta las sugerencias expresadas por compañeros y mantiene largos debates con el comité de redacción y, tras lo cual, efectúa su declaración de intenciones: se propone como meta ampliar su difusión, garantizar su calidad, mejorar el factor impacto, agilizar el proceso editorial, lograr una adaptación permanente al desarrollo de la especialidad ajustarla a la evolución y al progreso que sufren las revistas biomédicas, para lo cual no regateará esfuerzos ${ }^{16}$. Las modificaciones se evidencian ya en el primer número aparecido bajo su dirección en enero de 1995 [vol. XIX (1)], en la portada incluye, en su parte inferior derecha, una reproducción de un grabado histórico relacionado con la urología, motivo que mantendrá a lo largo de los 104 números que dirigirá en sus nueve años de permanencia en el puesto, por encima se encuentra el nombre de la revista y en el ángulo superior izquierdo el anagrama de la Asociación, de un tamaño más reducido que anteriormente, y sobre dos gruesas líneas en azul que paulatinamente se difuminan (Fig. 11), da un color sepia a los epígrafes y a los logotipos de las páginas informativas del principio le para resaltarlos; coloca debajo del título de los artículos el nombre de los autores y las palabras clave en español y en inglés, así como el número del volumen, el del mes de la publicación y el de las páginas que le corresponden. Se preocupa de que se realice la revisión sistemática de todos los manuscritos remitidos para su valoración por el comité de expertos; con la finalidad de facilitar la lectura cambia la tipografia y mejora las técnicas 


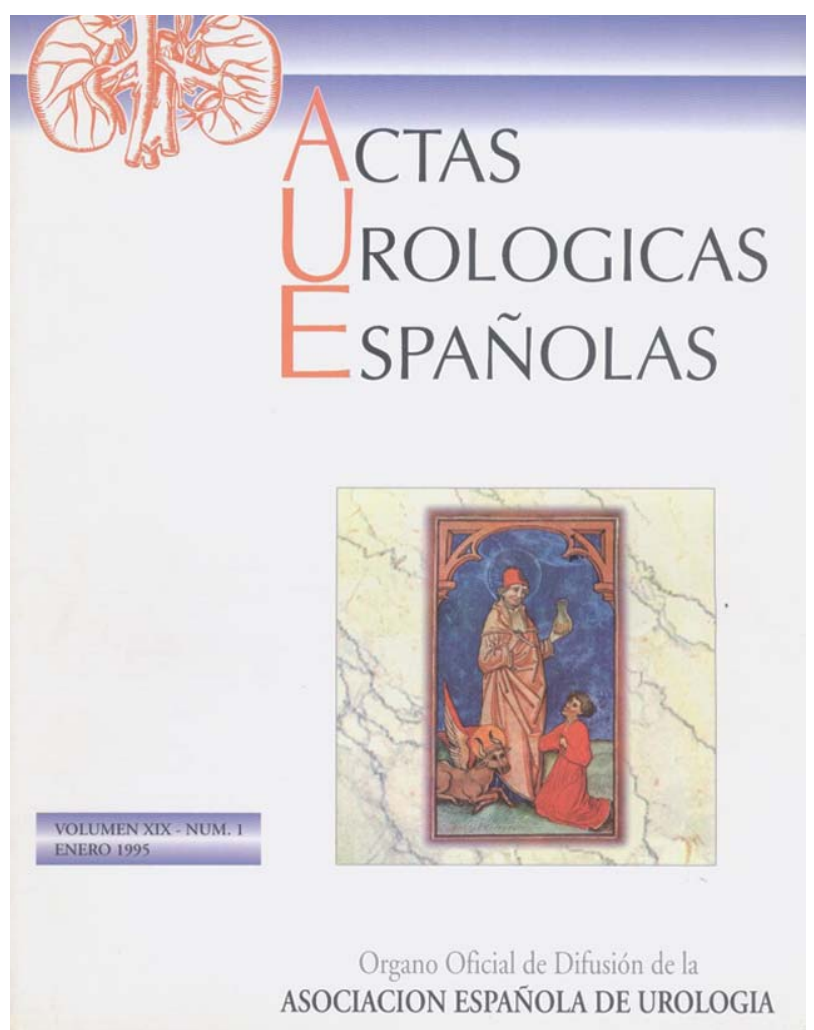

FIGURA 11. Portada del primer número modificado por el Dr. Carballido, enero de 1995, vol. XIX (1).

de reproducción, adapta las normas de presentación de originales a los requisitos técnicos propuestos en Vancouver, incrementa de modo notable el apartado de noticias y calendario y sustituye el índice general de autores y materias, publicado en el último número del año, desde 1995, por el presentado bajo la dirección del Dr. Alférez, desdoblado en el de materias y en el de autores colocados por orden alfabético [vol. XIX (10)], realizado en el Instituto de Estudios Documentales e Históricos sobre la Ciencia de Bibliometría y Análisis Documental, centro que, en 1999, se denomina Instituto de la Ciencia y Documentación López Piñero, con sede en Valencia.

En el ánimo del Dr. Carballido está el mantener con los lectores una continua comunicación acerca de los proyectos y de la evolución en la publicación, así un año después, en mayo de 1996 [vol. XX (5)], informa de las novedades que se van a introducir como son: mantener un editorial por número, presentar trabajos de investigación, crear una sección de imágenes en Urología, añadir el contenido de una conferencia clínica, de las impartidas en cursos, sesiones hospitalarias, trabajos del grupo de urólogos en formación, etc. y, por último, incorporar las citas bibliográficas por orden de aparición en el texto $^{17}$. Edita en 1966 el Directorio de los miembros de la $\mathrm{AEU}$, que se entrega en el congreso nacional celebrado en Santander, encuadernado con tapas de cartón duro en el que, además de recoger el registro de todos los asociados nacionales y extranjeros con sus nombres, dirección y centro de trabajo, incorpora año tras año informaciones útiles que lo enriquecen; en éste incorpora el nuevo reglamento aprobado en Madrid, el 3 de noviembre de 1995, en el que se señala que el cargo de director adjunto recaerá sobre el vocal de actividades científicas de la AEU (ex oficio) y que en el comité asesor figurarán los coordinadores de los grupos de trabajo; también la relación de los servicios de Urología y la de los hospitales españoles, con su ubicación, la abreviatura de las revistas médicas, el texto completo de las recomendaciones para redactar textos científicos y requisitos de uniformidad para presentar los originales, establecido en Vancouver en 1978 por un grupo de directores revistas biomédicas o normas de publicación de Vancouver y el Real Decreto sobre ensayos clínicos ${ }^{18}$; este modelo con variada información de interés general proseguirá en años sucesivos.

El Dr. Fariña remite una carta al director, en enero de 1996, en la que alaba su decisión de incluir en la portada motivos históricos urológicos y critica el poco cuidado que tienen los autores en respetar la ortografia de los nombres de personajes clásicos de la Medicina ${ }^{19}$; en el mes siguiente analiza, en un artículo, el número de autores por trabajo de los aparecidos en Actas Urológicas en un periodo de cuatro años y encuentra que en el $50 \%$ de los originales figuran más de seis firmantes, lo que excede lo aconsejado por las normas editoriales, en ambos casos solicita un mayor y más riguroso control de los textos por los revisores ${ }^{20}$.

En la asamblea general de la AEU, celebrada en Valladolid el 19 de junio de 1997, en el transcurso del LXII Congreso Nacional, en el informe anual que realiza el director de Actas Urológicas manifiesta que, durante los últimos tres años, el número de los trabajos recibidos es muy homo- 
géneo 179,170 y 178, y que en una revisión muy estricta aparecida en Medicina Clínica se cita a Actas Urológicas, junto con el Journal americano, como las revistas urológicas más sobresalientes $^{21}$. En el Directorio de 1997 señala la favorable acogida que ha tenido su edición con la cual quiere contribuir a mejorar el nivel de comunicación de todos los profesionales vinculados a la Urología, en este volumen aporta como datos de interés la Declaración de Helsinki de la Asociación Médica Mundial, una relación internacional de Asociaciones de Urología y direcciones urológicas útiles en Internet ${ }^{22}$.

En el Directorio de 1998 expone los cambios adoptados en el reglamento y aprobados en la asamblea de 20 de febrero y ratificados en la de 16 de junio del año en curso y en el que se modifica la composición de la comisión coordinadora que queda compuesta por: un comité editorial de seis personas, un consejo de redacción de doce y un consejo asesor de veinticuatro; desaparecen el cargo de vicepresidente y tres vocales y se introduce al tesorero de la AEU; el director de la revista asistirá a las reuniones de la junta directiva de la Asociación con voz pero sin voto y comunicará a la asamblea general, desarrollada en el congreso nacional, el estado de la revista. Añade en el volumen el informe internacional sobre Biomedicina y Ética del Consejo de Europa, UNESCO y $\mathrm{ONU}^{23}$.

En el LXIII congreso de Cádiz en junio de 1998, en su participación reglamentaria, indica que se mantiene estable la aportación de originales y que en el proceso editorial, desde su recepción a la publicación, transcurren alrededor de siete meses $^{24}$. Previa al congreso se distribuye una separata con las palabras clave para ser usadas en las comunicaciones al nacional, que, a partir de mayo de 2000, se incluirá cada año en el Boletín Informativo de la AEU. En el LXIV congreso, en Zaragoza, el 25 de mayo de 1999, señala en la asamblea general que el tiempo para la edición de un original ha disminuido y se encuentra entre mes y medio y seis meses, realiza unas reflexiones de cómo impulsar la revista que cifra en citarla más, aumentar las publicaciones en castellano y fomentar la realización de artículos de revisión ${ }^{25}$; en el Directorio entregado en ese año añade la dirección de organizaciones de enfermedades renales y urológicas y datos sobre Medicina y Bioética de la Organización Médica Colegial, Consejo de Europa y Asociación Médica Mundial ${ }^{26}$.

En junio de 2000 en el transcurso del LXV Congreso Nacional celebrado en Madrid, el director de Actas Urológicas, en el informe anual, apunta que se ha procedido a la renovación de los miembros de los comités y que desde el mes de enero se ha modificado el lomo de la revista con la inclusión del logotipo de la AEU, para que sea más fácil su identificación, noticia que detalla y amplía en dos editoriales sobre el desafio que representa un nuevo año y la labor directiva ${ }^{27-29}$; en el Directorio del año 2000, adjunta como ayuda adicional un diccionario inglés-español de términos informáticos y de Internet, así como direcciones de otras Asociaciones científicas ${ }^{30}$.

Se cumple en el 2001 el 25 aniversario de su edición, con este motivo su director ensalza la trayectoria mantenida por la publicación ${ }^{31}$ e inserta en la portada de los números el anagrama de 25 años (Fig. 12). El Dr. Cortiñas efectúa un

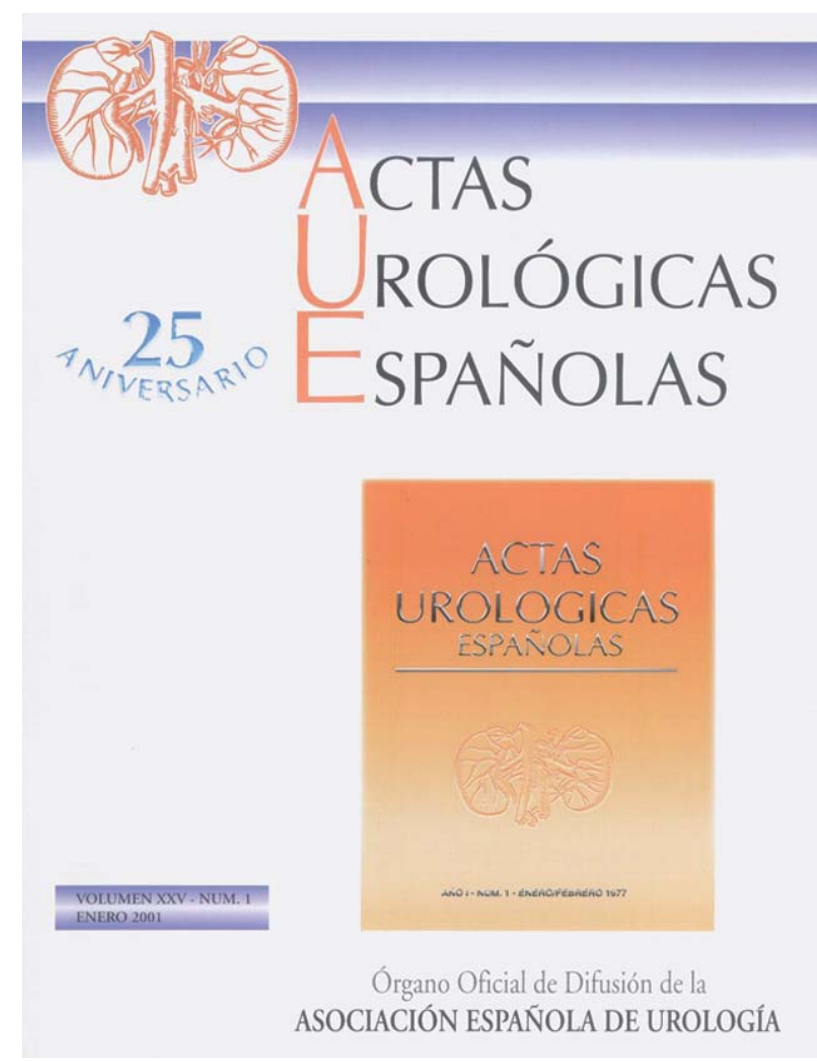

FIGURA 12. Portada del 25 aniversario de la publicación, 2001. 
estudio de la evolución que ha llevado la revista en sus veinte primeros años hasta 1996 ${ }^{32}$; el trabajo, resumen de su tesis doctoral, de calidad y buena hechura, aborda multitud de datos, entre otros, el número de páginas de cada volumen, el de las que están dedicadas a publicidad y sus porcentajes, la cantidad de artículos editados, desglosa a qué sección corresponden y extrae el total de cada uno de ellos; añade el nombre de los autores con mayor número de originales y el de de firmantes de cada uno de ellos, los temas tratados con mayor frecuencia, así como las palabras clave utilizadas $\mathrm{y}$, por último, la composición de los comités de redacción y asesor, todo ello acompañado de su correspondiente análisis y comentario; lamentablemente contiene una introducción histórica sobre el desarrollo de la AEU con escasa heurística y, por consiguiente, pobre información y abundantes errores que el Dr. Maganto Pavón corrige en una carta al director $^{33}$

En el acta de la asamblea de 15 de mayo de 2001, celebrada en Granada en el XLVI Congreso Nacional, el Dr. Carballido notifica que existe un buen equilibrio entre el número de trabajos que se recibe y el que se publica y también, de la inclusión de la revista en Internet, Actas on line, de tal forma que al mes de su edición se puede acceder al contenido de los principales artículos íntegros en la red ${ }^{34}$; en el Directorio de la AEU, repartido en el mismo congreso, se encuentran recomendaciones para escribir textos científicos y diversas unidades de medida en español e inglés ${ }^{35}$. Redacta, posteriormente, unas reflexiones acerca de las publicaciones en español, de su factor de impacto y sobre los índices de medida anglosajones así como de la importancia que aún mantienen trabajos en otras lenguas distintas al inglés y recuerda la preponderancia que cada vez más adquiere nuestro idioma; anuncia, además, un acuerdo de colaboración con la European Association of Urology (EAU) (Fig. 13), que se verá hecho realidad al final del año, gracias al cual se efectuará la publicación simultánea en Actas Urológicas y en European Urology, en inglés, de los mejores artículos científicos enviados a Actas Urológicas y seleccionados por los respectivos comités ${ }^{36,37}$.

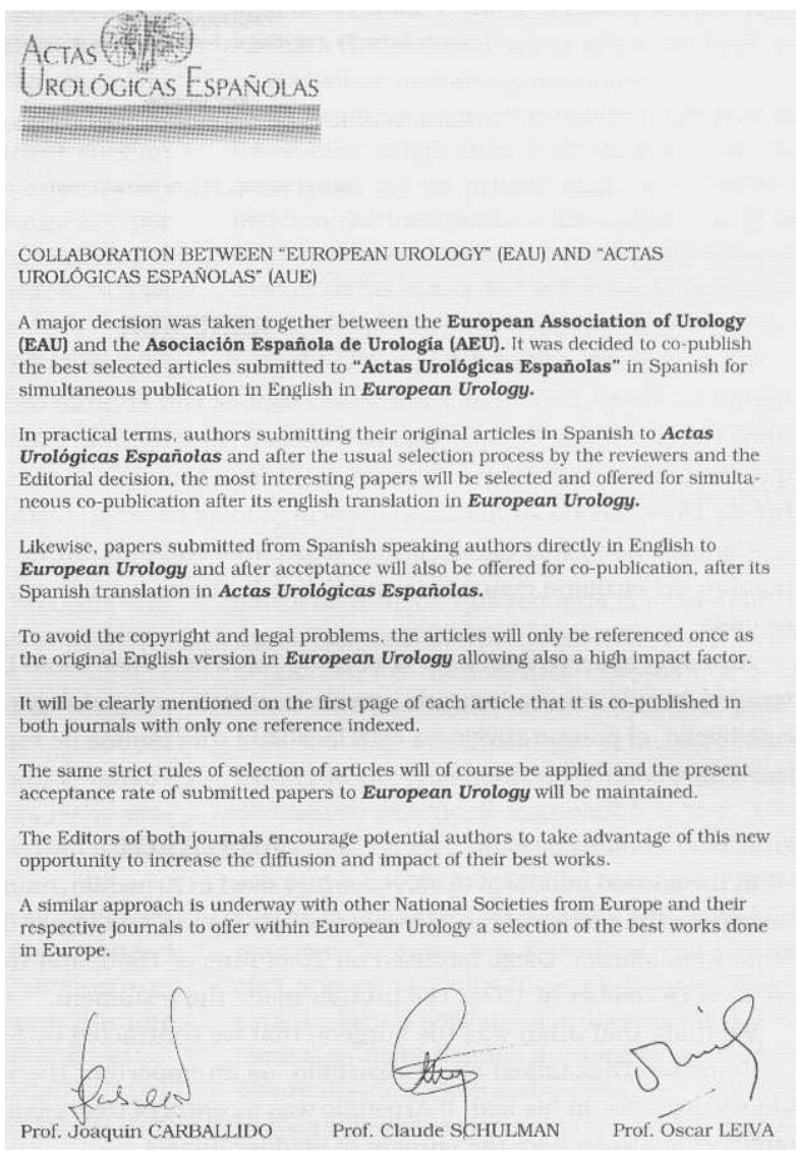

FIGURA 13. Acuerdo de colaboración con la European Urology. Vol. XXV (10).

Continúa el Dr. Carballido en junio de 2002 preocupado por la relevancia de las actividades científicas y sus órganos de medida y evaluación, especialmente en relación con la investigación biomédica, dominada por lo general por el Science Citation Index (SCI); como responsable de la publicación busca potenciar la contribución científica escrita en castellano y logra la inclusión de Actas Urológicas en el Índice Bibliográfico Español en Ciencias de la Salud (IBECS), del Instituto de la Salud Carlos III, como centro coordinador en España del proyecto para el desarrollo de la biblioteca virtual de Salud (BVS) en colaboración con la Biblioteca Regional de Medicina (BIREME) de la Organización Panamericana de la Salud, de la Organización Mundial de la Salud (OPS/OMS) ${ }^{(38)}$. En el informe anual que presenta el director, en el Congreso Nacional desarrollado en Murcia el 7 de mayo de 2002, abunda en lo anterior: que su inclusión en el IBEX de la 
Biblioteca Nacional de Salud significa que, de las 500 revistas editadas en España, Actas Urológicas es una de las 115 seleccionadas; añade que el factor impacto de la publicación se encuentra en el $0,25 \%$, que las secciones con que cuenta se encuentran muy consolidadas y que se crea una nueva de Historia de la Urologia; en el Directorio entregado en el congreso, aporta una relación de direcciones urológicas en Internet ${ }^{39}$.

Como continuación de la efeméride que representa el cuarto de siglo de la publicación, en el número de julio / agosto de 2002 [vol. XXVI (7)], la Dirección y la Redacción solicitan a diversos profesionales de "acreditada experiencia, excelencia académica, práctica clínica y compromiso editorial con Actas Urológicas" artículos de colaboración que reflejen de la manera más acertada y reflexiva posible la visión de presente y la predicción de futuro de los temas más relevante que conforman la Urología, los cuales aparecerán con un logotipo característico en el volumen $\mathrm{XXVI}^{40}$, recogidos entre los números 7 a 10 de 2002 .

En febrero de 2003 el Dr. Fernández Aparicio y colaboradores escriben un interesante y bien preparado artículo acerca de los análisis estadísticos de los originales publicados en Actas Urológicas, tras el cual indican la necesidad de profundizar en el conocimiento de las técnicas estadísticas para su correcta valoración ya que una gran cantidad de ellos incorpora estudios complejos en su desarrollo ${ }^{41}$. El Dr. Millán Rodríguez puntualiza y corrige algunos puntos del trabajo, que considera de interés ${ }^{42}$.

En su última intervención, en la asamblea del LXVIII Congreso Nacional celebrado en Las Palmas, el 27 de mayo de 2003, el Dr. Carballido expone que mensualmente se reciben entre $14 \mathrm{y}$ 16 originales; que por el hecho de estar introducido en SCIELO, Scientific electronic library on line, proyecto estrella de la OMS que identifica a las revistas de excelencia, se incrementa el valor impacto de la publicación un $150 \%$, por lo que considera que, desde el punto de vista bibliométrico, Actas Urológicas es una publicación consolidada y competitiva dentro de las de habla hispana $^{43}$. El Directorio de 2003 incorpora la ley sobre derechos y obligaciones del paciente y publicaciones y asociaciones de Urología interna- cionales ${ }^{44}$. Su despedida la efectúa con un editorial que titula La hora del relevo (Fig. 14), en el que, tras repasar la posición lograda por la revista, señala algunos de los retos que le quedan aún por alcanzar ${ }^{45}$.

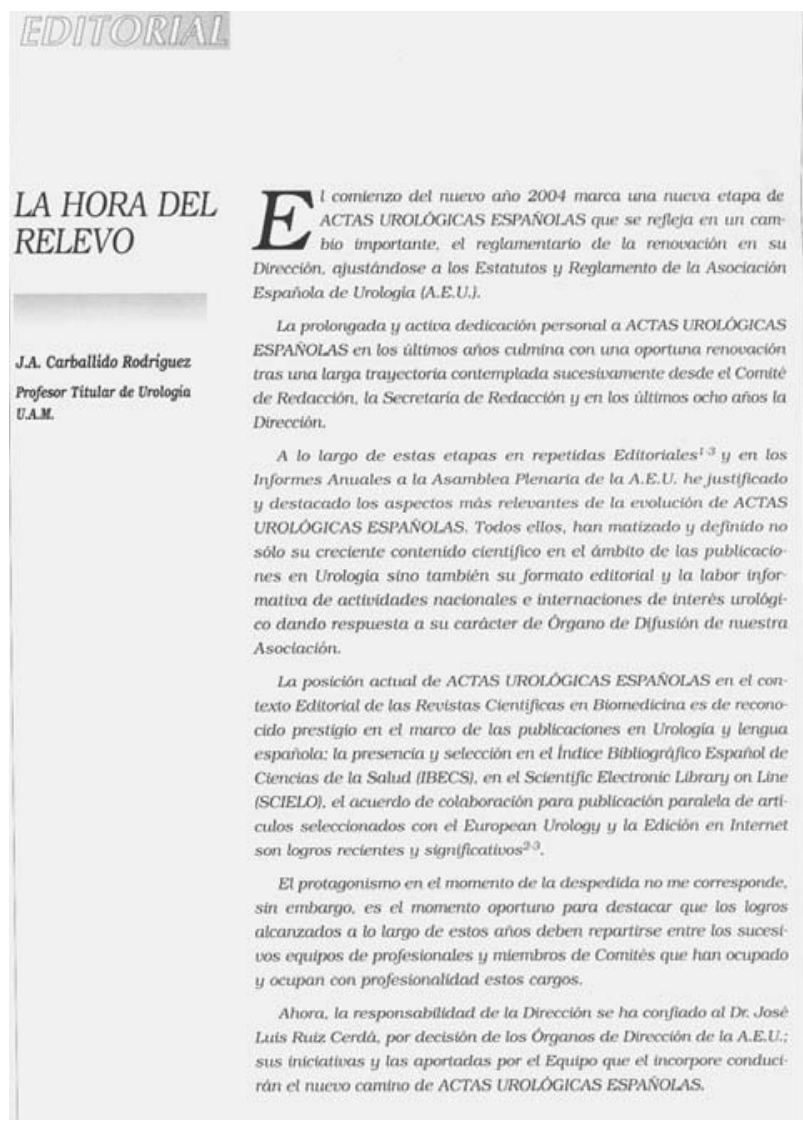

FIGURA 14. Despedida del Dr. Carballido, La hora del relevo, enero de 2004, vol. XXVIII (1).

\section{Dirección del Dr. D. José Luis Ruiz Cerdá (diciembre 2003). Vol. XXVIII (1)}

Elegido como director en diciembre de 2003 y, como adjunto, el vocal de actividades científicas, Dr. Carlos Llorente Abarca, comienza su andadura en enero de 2004, cifra su propósito en mejorar la publicación en su doble vertiente, como órgano de expresión de la AEU y como herramienta científica útil y práctica, se propone para ello incrementar su nivel de impacto e incluirla en el Journal Citation Index, para lograr consolidarla como la primera revista de habla hispana en Urología $^{46}$. En marzo de 2004 [vol. XXVIII (3)] ya se pone en evidencia su cambio en la portada, en la que, con análogo formato, en lugar de recuadro 
con motivo histórico aparecen los títulos de los artículos del número, uno de los cuales resalta como "lectura recomendada", y añade a pie de página la dirección en Internet de Actas Urológicas (Fig. 15). Además, se realizan otros cambios en la edición como la creación de un guión por materias, adaptación de la bibliografía a las normas internacionales y modificación de la edición de la información sobre eventos científi$\cos$ a un formato uniforme y estandarizado.

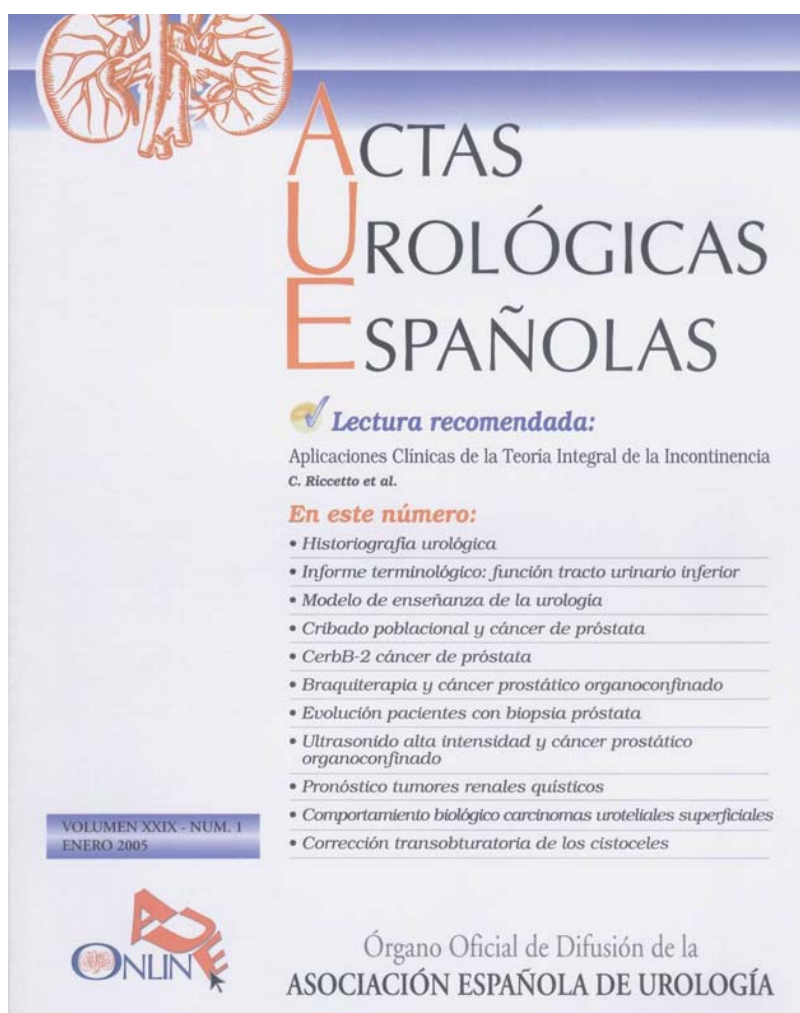

FIGURA 15. Portada con las modificaciones del Dr. Ruiz Cerdá, enero de 2004, vol. XXVIII (1).

En el LXIX Congreso Nacional de Oviedo, el 8 de junio de 2004, tiene lugar una asamblea general extraordinaria de la AEU, en la que se discute una propuesta de la junta directiva referente a la modificación de Actas Urológicas Españolas en el sentido de unificar las revistas urológicas editadas en español y en portugués, como la Panamericana, la de la Confederación Americana (CAU), la Portuguesa, la Brasileña y posiblemente alguna más, idea considerada buena y ambiciosa por los proponentes con vistas a lograr una mayor difusión e incrementar el factor impacto de la publicación y, de este modo, tener una mayor valoración científica internacional. Ello da lugar a un amplio debate en el que se escuchan múltiples opiniones, la mayoría adversas, en primer lugar, porque necesita de un estudio y valoración que no están hechos, en segundo, porque es una noticia que ha producido sorpresa en los socios por lo nueva e inesperada y precisa ser analizada en profundidad para ver las ventajas y los inconvenientes que tiene $y$, por último, porque es preciso conocer lo que desean los urólogos españoles: poder publicar los trabajos como hasta ahora o hacerlo en menor número pero con mayor calidad y valor científico, por todo ello la junta retira la proposición por inmadura y el Dr. Ruiz Cerdá, como director y dada la importancia del tema sugiere dejar un periodo de reflexión y que todos los que tengan alternativas aporten sugerencias sobre el modo de aumentar el factor de impacto; en la asamblea ordinaria que tiene lugar a continuación agradece la confianza puesta en él para dirigir la revista, indica el interés que ésta despierta a tenor de la discusión anterior, añade que independientemente de que se lleve a cabo la fusión o no, lo importante es incrementar el nivel científico, motivo por el que va a trabajar y señala algunos cambios menores que va a efectuar en la edición ${ }^{47}$.

En el Congreso de 2004 y editados por el Dr. Ruiz Cerdá, se entregan los volúmenes extraordinarios, tanto el libro de resúmenes de las comunicaciones presentadas como del Directorio de la AEU, que cumple en éste su décima edición e incorpora el Convenio europeo sobre Derechos Humanos y la Biomedicina, ambos mantienen análogas características a los que los precedieron $^{48}$.

El primero de Marzo del 2005 se crea la pagina web oficial de Actas Urológicas Españolas impulsada por el Dr. Ruiz Cerdá junto a Ene Ediciones S.L. con la dirección www.actasurologicas.info. Mediante esta página, se modifica toda la gestión del proceso de recepción de trabajos y de la comunicación entre director de la revista y los revisores que, a partir de entonces, se puede realizar integramente on line. Además, para obtener información del rendimiento de la página web, se contrata un servicio externo de gestión 
del servidor a través de la empresa Urchin 5. Esto permite obtener información relevante sobre determinados indicadores como el número de visitas media al mes, el número de descargas de trabajos y conocer el origen de donde proceden los usuarios que consultan la página web. Estos indicadores aportan información directa, por primera vez, del interés que despierta la revista a nivel nacional e internacional. Desde entonces se viene publicando una estadística resumida de actividad de actasurologicas.info en el boletín informativo trimestral de la AEU, así como una relación de los diez trabajos más descargados.

$\mathrm{El}$ informe del director de Actas en la asamblea del LXX Congreso Nacional de San Sebastián, el 7 de junio de 2005, recoge las novedades que ha llevado a cabo, ha pedido permiso para traducir diversas guías clínicas y editarlas en Actas Urológicas, ha solicitado su colaboración a diversos expertos nacionales y extranjeros y a los autores de algunos de los trabajos presentado en el Congreso Nacional para su publicación, la cantidad de originales por número sube a diez y va a editar varios monográficos ${ }^{(49)}$; el Directorio de los socios de la AEU del año 2005 contiene, además, la Ley de ordenación de los profesionales sanitarios, de 6 de noviembre de $2003^{50}$. Además, se informa de la ampliación de comité de redacción, la creación de un comité específico para la revisión metodológica de los trabajos y la realización de la primera reunión durante el congreso del comité de redacción a la que acudieron un número importante de revisores. En esta reunión, se realizó una puesta al día de las inquietudes de los revisores y del director.

En mayo de 2006, y editado por el Dr. R. Vela Navarrete aparece un número monográfico dedicado a la memoria del Dr. D. Luis Cifuentes Delatte, con motivo de su fallecimiento y otro en el mes de mayo sobre aprendizaje en Laparoscopia, dirigido por los Drs. J. Amón Sesmero, C. Hernández Fernández y C. Rioja Sanz [XXX (5)]. En el de junio de 2006, nos informa el director de la mecánica que se sigue en el tratamiento de los originales y de la tarea del revisor de artículos, su labor como editor tras recoger el manuscrito es la de aconsejar las modificaciones que le proponen y tomar la decisión de publicarlo o no, para ello se rige por las normas metodológicas de la medicina basada en la evidencia, éticas y de publicación y considera que la labor del revisor es esencial ${ }^{51}$.

En el LXXI Congreso Nacional celebrado en Sevilla, en la asamblea general el 13 de junio de 2006, el presidente de la AEU, Dr. Castiñeiras, informa que la junta directiva ha tomado el acuerdo de traducir al inglés los artículos de la revista que aparezcan en Internet, de tal modo que la publicación en papel no sufrirá modificación alguna. Por lo que, desde Enero del 2006, la revista se convierte en bilingüe español-inglés. Si bien la versión inglés se encuentra únicamente en la versión on line de la página web. Esta decisión intenta romper la barrera idiomática que impide la obtención de un mayor factor impacto. La medida supone un gran esfuerzo económico por parte de la AEU. Puesto que, la traducción de los artículos correrá a cargo de los presupuestos de ésta. A partir de entonces, todos los artículos que sean aceptados para su publicación en la revista serán traducidos al inglés y "colgados" en actasurologicas.info. Como medidas complementarias a esta importante decisión, se inician los trámites para lograr que la revista aparezca como de libre acceso (free access) y como bilingüe en las búsquedas bibliográficas realizadas mediante pubmed. Al mismo tiempo, se solicita un link directo entre las búsquedas en pubmed y el servidor de actasurologicas.info.

Edita, también, como presidente de la Asociación, con el logotipo de Actas Urológicas, el reglamento de la AEU en formato de bolsillo, de fácil manejo y para que todos los miembros puedan consultarlo, y en el que se incluye la foto de todos los presidentes que hasta la actualidad ha tenido la Asociación Española de Urología.

En el Directorio del año 2006 el Dr. Ruiz Cerdá resalta en su presentación el valor que tiene tanto histórico y demográfico urológico como de utilidad práctica ya que es un registro dinámico que se actualiza cada año; añade como información suplementaria la Estrategia Sanitaria de la Comunidad Europea ${ }^{52}$.

\section{COMENTARIOS}

Tras la aprobación en julio de 1976 de la puesta en marcha de Actas Urológicas Españolas, se logra, después de sesenta y cinco años, desde 
la fundación de la Asociación Española de Urología en 1911, y luego de tres cortos periodos en los que tuvo su órgano de representación oficial en diversas publicaciones ${ }^{53-54}$, cumplir el objetivo institucional de disponer de un medio propio de difusión. Regido por una comisión coordinadora cuya actual composición, especificada en la los estatutos, se encuentra compuesta por la junta permanente de la Asociación, el director de la revista, un miembro del comité de redacción y otro del asesor, las amplias atribuciones otorgadas a su director hacen que sea su persona la responsable de su marcha, de ahí que hayamos considerado lo más conveniente repasar la actividad realizada por cada uno de los que ha tenido la publicación para conocer su evolución histórica.

De su primer director, el Dr. Alférez, señalamos que trabajó con intensidad, ilusión y eficacia para poner en marcha la revista desde la nada, dándole el formato, las principales secciones y las características generales que en esencia aún mantiene, con lo que logró dotar a la publicación de una categoría que aumentó la proyección nacional y el prestigio de la Asociación.

La actuación del Dr. Leiva en sus nueve años como director podemos definirla como de responsabilidad. Preocupado por la actualización de la publicación y por incrementar su calidad científica la potenció con una presentación adaptada a las directrices de las ediciones biomédicas con la finalidad de que tuviese un papel en el contexto internacional para lo que, además, la incluyó en los índices médicos de consulta; aumentó la edición anual para dar cabida al gran número de originales remitidos; estableció los premios a los mejores artículos editados en el año; imprimió las ponencias de los temas monográficos del Congreso Nacional y le dio un formato estandarizado con el logotipo de la revista, además de editar el Directorio de los miembros de la AEU como un número especial de Actas Urológicas $\mathrm{y}$, a pesar de todos estos cambios, mantuvo su autofinanciación. Destacamos su manifiesto interés por consolidarla como referente de la Urología española y su extrema gratitud hacia todos los colaboradores que participaron en la consecución de los objetivos propuestos.

La labor del Dr. Carballido se puede definir de comunicativa y de escrupulosa con la información, marcada por el compromiso que adquirió al aceptar el puesto. En su larga vinculación con la revista, desde mayo de 1985, su inicial etapa como miembro del comité de redacción, seguido en 1989 de su periodo de secretario de redacción hasta el final de su mandato directivo, ha sido un luchador sin descanso y sin desánimo, ha mostrado una activa e intensa dedicación a mejorar su calidad científica, a potenciar su difusión al aumentar su inclusión en los índices bibliográficos, al introducirla en Internet y al establecer relaciones con la European Urology, para, de este modo, situarla en un alto contexto internacional y elevar su índice de impacto; mejoró el Directorio anual al incorporar en él información de interés urológico y médico general y, finalmente, ha sido su valedor en defensa de su primacía como la publicación urológica de referencia en español.

El Dr. Ruiz Cerdá, en su corta etapa, es consciente de que, para elevar el nivel científico, es preciso aumentar la calidad de los originales y considera que para ello, además de concienciarse sus autores, el papel de los revisores es fundamental para conseguir este objetivo. Por último, la aparición de Actas on Line, traducida al inglés, es un exponente de la preocupación de la junta coordinadora para que el trabajo científico de los españoles tenga su reconocimiento internacional.

Como colofón de este repaso histórico añadimos que, con la celebración de este aniversario, se demuestra la consolidación que la publicación ha logrado, que se ha situado en un nivel predominante del panorama urológico hispano, con un alto índice de aceptación nacional. La preocupación por una mayor difusión internacional es la tarea dominante para que su factor impacto sea cada vez más relevante, todos somos conscientes de que ello pasa por incrementar la calidad de nuestras aportaciones y por que citemos un mayor número de veces a Actas Urológicas Españolas, nuestra revista, puntual referencia para publicar en castellano y soporte de difusión de la Asociación Española de Urología, magistralmente regida, gracias a la labor, entrega y desinteresada contribución de sus directores.

Recogemos en Tabla 1 la relación de directores y en la Tabla 2 la de los directores adjuntos que ha tenido la revista desde su creación hasta la actualidad y en la Tabla 3 los hechos más relevantes de todo el periodo. 
Tabla 1

\begin{tabular}{lcccc}
\hline & Nombramiento & Primer Número & Números editados & Último número \\
\hline Alférez & 6 de julio de 1976 & Enero de 1977 & 57 & Marzo/abril 1986 \\
Leiva & 12 de mayo de 1985 & Mayo/junio de 1986 & 88 & Nov./dic. 1994 \\
Carballido & 1 de octubre de 1994 & Enero de 1995 & 90 & Nov./ dic. 2003 \\
Ruiz cerdá & Diciembre de 2003 & Enero de 2004 & 29 & \\
\hline
\end{tabular}

\section{Tabla 2}

\section{Directores adjuntos:}

Dr. D. Francisco Javier Solé Balcells.

Dr. D. José Luis Insausti Cordón.

Dr. D. Óscar Leiva Galvis.

Dr. D. Narciso Serrallach Mauri.

Dr. D. Joaquín Carballido (en funciones).

Dr. D. Marceliano García Pérez.

Dr. D. Carlos Rioja Sanz.

Dr. D. David Castro Díaz.

Dr. D. Carlos Llorente Abarca.
Julio de 1976 a mayo de 1982

Mayo de 1982 a diciembre de 1985

Enero 1986 a abril de 1986

Mayo 1986 a enero 1987 (Vacante)

Enero de 1987 a abril de 1994

Mayo de 1994 a diciembre de 1994

Enero de 1995 a junio de 1997

Julio 1997 a mayo de 2001

Junio de 2001 a mayo de 2005

Junio de 2005

\section{Tabla 3}

Acontecimientos más notables de estos 30 años

6 de julio de 1976. Aprobación de la puesta en marcha de la revista.

Enero de $1977 . \quad$ Edición del primer número.

1987.

Establecimiento de los premios de Actas al mejor artículo publicad en el año.

Marzo/abril 1989.

Se adapta a las normas internacionales la admisión de originales.

Junio de 1990.

Publicación del libro de Resúmenes de las comunicaciones al congreso nacional.

Junio de 1991.

Se imprimen las ponencias del tema monográfico del Congreso Nacional.

Enero de 1992.

Se amplia a diez números anuales, edición mensual, excepto agosto y diciembre.

Enero de 1992.

Se incluye en los índices de consulta de las publicaciones médicas. Excerta Médica, Índice Médico Español y Index Medicus.

Junio de 1993. Comienza a editarse el Directorio y Reglamento de los miembros de la AEU.

Noviembre de 1995. Adaptación de originales a las normas de Vancouver.

Noviembre de 1995. El cargo de director adjunto recae en el vocal de Actividades Científicas.

Mayo de 2001.

Aparición de Actas on Line.

Diciembre de 2001.

Acuerdo de colaboración con European Urology.

Junio de 2002.

Inclusión en el IBCS y en el IBEX.

Marzo de 2005

Creación de la página web: actasurologicas.info

2006.

Actas on Line en inglés. 


\section{REFERENCIAS}

1. Reglamento. Actas Urol. Esp. 1987;11(2)

2. Editorial. Actas Urol. Esp. 1977;I(1).

3. Alférez Villalobos C. Editorial. Actas Urol. Esp. 1985;(1);1.

4. Editorial. Actas Urol. Esp. 1987;11(2);1-2.

5. Premios de Actas Urológicas, núm. Monográfico, Infección urinaria. Actas Urol. Esp. 1988.

6. Pérez Albacete M. Congresos celebrados por la AEU en el siglo XX. Urol. Integr. Invest. 2002;7(2):205-212.

7. Boletín Informativo de la AEU, nº 10, enero de 1991.

8. Leiva Galvis O. Carta al director. Actas Urol. Esp. 1991;15 (5).

9. Vela Navarrete R. Presentación. Actas Urol. Esp. Junio, 1993; núm. monográfico.

10. Leiva Galvis O, Carballido Rodríguez J. Reglamento y directorio de la Asociación Española de Urología. Actas Urol. Esp. Junio, 1993; núm. monográfico.

11. Blasco Casares FJ, Pinsach Elías Ll. Análisis crítico de la bibliografía de Actas Urológicas Españolas. Critical analysis of the bibliography of Actas Urologicas Espanolas Actas Urol. Esp. 1993;17(6):347-350.

12. Medios de Comunicación y ética. Actas Urol. Esp. 1994; 18 (4).

13. Leiva Galvis O. El deber cumplido. Actas Urol. Esp. Mayo, 1994; XVIII, núm. monográfico: 333.

14. Carballido Rodríguez J, Leiva Galvis O. cifras y datos. Actas Urologicas Espanolas: figures and data] Actas Urol. Esp. 1994 May;18 Suppl:334-336

15. Boletín Informativo de la AEU, n 26, marzo de 1995.

16. Carballido Rodríguez J. Comites de ëtica en investigación. Buen presente y mejor futuro. Ethical committees of clinical research: Good present. Better future?. Actas Urol. Esp. 1995;19(8):595-598.

17. Carballido Rodríguez J. Crónica de un proceso: la evolución de Actas Urológicas Españolas. Chronicle of a process: the evolution of Actas Urologicas Espanolas Actas Urol. Esp. 1996;20(5):399-401.

18. Reglamento, directorio y...: su razón de ser. Reglamento y Directorio de la AEU. Actas Urol. Esp. 1996.

19. Fariña LA. Actas Urológicas Españolas, la historia y la ortografia. [Actas Urologicas Espanolas, history and spelling] Actas Urol. Esp. 1996;20(1):85.

20. Fariña LA. Número de autores en Actas Urológicas Españolas. Number of authors in Actas Urologicas Espanolas] Actas Urol. Esp. 1996;20(2):195-198.

21. Boletín Informativo de la AEU, nº 40, mayo de 1998.

22. Directorio de la AEU. Actas Urol. Esp. 1997.

23. Nuevo Reglamento y Directorio de la AEU. Actas Urol. Esp. 1998.

24. Boletín Informativo de la AEU, nº 44, abril de 1999.

25. Boletín Informativo de la AEU, nº 48, abril de 2000.

26. Directorio de la AEU. Actas Urol. Esp. 1999.

27. Boletín Informativo de la AEU, nº 52, abril de 2001.

28. Carballido Rodríguez J. Continúa el desafío. Actas Urol. Esp. 2000; 14 (1):1-2.

29. Carballido Rodríguez J. Gratitud y desafios (Nuevos retos). Actas Urol. Esp. 2000;14(6):444-445.

30. Directorio de la AEU. Actas Urol. Esp. 2000.

31. Carballido Rodríguez J. 25 Aniversario de Actas Urológicas Españolas. Actas Urol. Esp. 2001;15(1):1-2.

32. Cortiñas González JR. Los primeros 25 años de Actas Urológicas Españolas (1977-1996). [The first 20 years of history in Actas Urologicas Espanolas (1977-1996)] Actas Urol. Esp. 2001;15(1):3-13.
33. Maganto Pavón E. Algunas puntualizaciones sobre "Los primeros 25 años de Actas Urológicas Españolas (19771996)" On "The first 20 years of history of Actas Urologicas Espanolas (1977-1996)”]. Actas Urol. Esp. 2001;15(3):248.

34. Boletín Informativo de la AEU, nº 56, abril de 2002.

35. Nuevo Reglamento y Directorio de la AEU. Actas Urol. Esp. 2001.

36. Carballido Rodríguez J. Reflexiones editoriales las publicaciones en español. reflections on publications in Spanish] Actas Urol. Esp. 2001;15(9):607-609.

37. Leiva Galvis O, Carballido Rodríguez J. Carta del presidente de la AEU y del director de la AUE. Actas Urol. Esp. 2001;15(10):691.

38. Carballido Rodríguez J. Índice bibliográfico español en Ciencias de la Salud (IBECS) y Actas Urológicas Españolas. The Spanish Bibliographic Index of the Health Sciences (IBECS) and Actas Urologicas Espanolas Actas Urol. Esp. 2002;16(6):381-383.

39. Boletín Informativo de la AEU, n 60, abril de 2003.

40. Docencia, investigación y asistencia en Urología: años a través de Actas Urológicas Españolas. Actas Urol. Esp. 2002;17(7):443-444.

41. Fernández Aparicio T, Miñana López B, Guzmán MartínezValls P, Hita Villaplana, G. Uso de los análisis estadísticos en los artículos originales de Actas Urológicas Españolas. Accesibilidad para el lector. Actas Urol. Esp[Use of statistical analysis in original articles in "Actas Urologicas Espanolas.” Accessibility for the reader]. 2003; 17(2):103109.

42. Millán Rodríguez F. Referencia al artículo "Uso de los análisis estadístico en los artículos originales de Actas Urológicas Españolas. Accesibilidad para el lector[In Reference to the article " Use of statistical analysis in original articles published in Actas Urologicas Espanolas: accessibility for the reader"]". Actas Urol. Esp. 2003;17(7): 566.

43. Boletín Informativo de la AEU, no 64, abril de 2004.

44. Directorio de la AEU. Actas Urol. Esp. 2003.

45. Carballido Rodríguez J. La hora del relevo. Actas Urol. Esp. 2004;18(1):1-2.

46. Ruiz Cerdá, JL. Editorial. Actas Urol. Esp. 2004;18(1):3-4.

47. Boletín Informativo de la AEU $2^{\underline{a}}$ época, $\mathrm{n}^{-2} 2$, mayo de 2005.

48. Directorio de la AEU. Actas Urol. Esp. 2004.

49. Boletín Informativo de la AEU (segunda serie), $\mathrm{n}^{\circ} 5$, mayo de 2006.

50. Nuevo Reglamento y Directorio de la AEU. Actas Urol Esp. 2005.

51. Ruiz Cerdá JL. La tarea del revisor de artículos para Actas Urológicas Españolas. Actas Urol Esp. 2006;30(6):555.

52. Directorio de la AEU. Actas Urol. Esp. 2006.

53. Vela Navarrete R, Insausti Cordón, JL. Aparición de Actas Urológicas Españolas. Actas Urol. Esp. 1977; I (3).177-179

54. Pérez Albacete, M. La actividad científica de la AEU recogida en las publicaciones periódicas. Scientific activities of the Spanish Urology Association gathered in periodic publicationsActas Urol. Esp. 2006 Mar;30(3):243-253.

Correspondencia autor: Dr. M. Pérez Albacete

Hospital Universitario Virgen de la Arrixaca

Ctra. Murcia-Cartagena, s/n. 30120 El Palmar (Murcia).

Tel.: 968369779

E-mail autor: mariano.perez2@carm.es

Información artículo: Historia de la Urología

Trabajo recibido: septiembre 2006

Trabajo aceptado: enero 2007 